Three-dimensional models of metal-poor stars

This content has been downloaded from IOPscience. Please scroll down to see the full text. 2008 Phys. Scr. 2008014004

(http://iopscience.iop.org/1402-4896/2008/T133/014004)

View the table of contents for this issue, or go to the journal homepage for more

Download details:

IP Address: 150.203.88.111

This content was downloaded on 26/06/2014 at 07:03

Please note that terms and conditions apply. 


\title{
Three-dimensional models of metal-poor stars
}

\author{
R Collet \\ Max-Planck-Institut für Astrophysik, Postfach 1317, D-85741 Garching, München, Germany \\ E-mail: remo@mpa-garching.mpg.de
}

Received 17 September 2008

Accepted for publication 19 September 2008

Published 19 December 2008

Online at stacks.iop.org/PhysScr/T133/014004

\begin{abstract}
I present here the main results of recent realistic, three-dimensional (3D), hydrodynamical simulations of convection at the surface of metal-poor red giant stars. I discuss the application of these convection simulations as time-dependent, 3D, hydrodynamical model atmospheres to spectral line formation calculations and abundance analyses. The impact of 3D models on derived elemental abundances is investigated by means of a differential comparison of the line strengths predicted in 3D under the assumption of local thermodynamic equilibrium (LTE) with the results of analogous line formation calculations performed with classical, 1D, hydrostatic model atmospheres. The low surface temperatures encountered in the upper photospheric layers of 3D model atmospheres of very metal-poor stars cause spectral lines of neutral metals and molecules to appear stronger in 3D than in $1 \mathrm{D}$ calculations. Hence, 3D elemental abundances derived from such lines are significantly lower than estimated by analyses with 1D models. In particular, differential 3D-1D LTE abundances for C, $\mathrm{N}$ and $\mathrm{O}$ derived from $\mathrm{CH}, \mathrm{NH}$ and $\mathrm{OH}$ lines are found to be in the range -0.5 to -1 dex. Large negative differential 3D-1D corrections to the Fe abundance are also computed for weak low-excitation Fe I lines. The application of metal-poor 3D models to the spectroscopic analysis of extremely iron-poor halo stars is discussed.
\end{abstract}

PACS numbers: 95.30.Lz, 97.10.Ex, 97.10.Tk

\section{Introduction}

The first evidence for the existence of stars characterized by a lower abundance of metals with respect to hydrogen than in the Sun came in the mid-20th century, in particular with the works of Schwarzschild and Schwarzschild (1950) and Roman (1950). These authors observed that giant and dwarf stars with weaker metal lines in their spectra typically had a larger velocity dispersion than stars with stronger spectral lines; also, high velocities appeared to be associated only with the weak-line stars. These works suggested that population II stars (as dubbed by Baade 1944) differed from population I stars not only by their kinematic properties but also in terms of basic chemical composition, the former being characterized by an overall lower iron abundance. In the following year, Chamberlain and Aller (1951) produced the first quantitative abundance analysis of population II stars; the authors analysed the two stars HD19445 and HD140283 and derived significantly lower abundances of calcium and iron with respect to hydrogen than in the Sun, which actually allowed explanation of the peculiar spectral and photometric properties of these objects.

It soon became clear that metal-poor stars were extremely interesting objects that could provide important clues about the formation and the chemical and dynamical evolution of the Galaxy. Better tools for quantitative spectroscopy of low-metallicity stars were therefore also required, and the first 1D model stellar atmospheres of metal-poor stars started to appear in the late 1960s to early 1970s. Noteworthy are the works by Krishna Swamy (1969) and Gustafsson et al (1975); the latter in particular constitutes the first extended grid of theoretical 1D model atmospheres of giant stars with metallicities ranging from solar down to $[\mathrm{Fe} / \mathrm{H}]=-3^{1}$. Since then, 1D model stellar atmospheres have been continuously

${ }^{1}[\mathrm{Fe} / \mathrm{H}] \equiv \log \left(n_{\mathrm{Fe}} / n_{\mathrm{H}}\right)-\log \left(n_{\mathrm{Fe}} / n_{\mathrm{H}}\right)_{\odot}$, where $n_{\mathrm{Fe}}$ and $n_{\mathrm{H}}$ are the number densities of $\mathrm{Fe}$ and $\mathrm{H}$, respectively, and the subscript $\odot$ symbol refers to the Sun. 
developed and improved (see, e.g., reviews by Asplund 2005 and Gustafsson and Jorgensen 1994), especially with regard to input physics and opacities, and are even now the most widely used models in stellar abundance analyses.

Yet, classical 1D model stellar atmospheres are normally constructed under a number of simplifying assumptions, such as plane-parallel geometry or spherical symmetry, hydrostatic equilibrium and flux constancy. Moreover, 1D modelling of stellar atmospheres inherently relies on rudimentary implementations of convective energy transport, which is usually treated by means of the mixing-length theory (Böhm-Vitense 1958) or similar alternative formulations (e.g. Canuto and Mazzitelli 1991), all characterized by a number of tunable but not necessarily physical free parameters. In late-type stars however, the convection zone reaches and appreciably influences the surface layers from which the emergent stellar flux is formed. For instance, high spatial resolution imaging of the solar photosphere immediately reveals a distinctive granulation pattern that reflects the bulk gas motions in the upper part of the convective zone near the optical surface. Given the dynamic and multidimensional character of convection, the use of time-independent, hydrostatic, 1D, model atmospheres in abundance analyses of late-type stars is arguably a potential source of severe systematic errors. In addition, the strengths and exact shapes of spectral lines cannot possibly be predicted in 1D without resorting to ad hoc fudge parameters such as micro- and macro-turbulence, which are supposed to account for non-thermal Doppler broadening due to bulk flows in the stellar atmosphere. During the past 30 years, however, various computer codes have been developed to perform time-dependent, 3D, hydrodynamical simulations of convection at the surface of late-type stars (e.g. Asplund et al 1999, Carlsson et al 2004, Freytag et al 2002, Ludwig et al 2002, Nordlund 1982, Nordlund and Dravins 1990, Stein and Nordlund 1998, Vögler 2004). Such stellar surface convection simulations can naturally be used as time sequences of $3 \mathrm{D}$ model stellar atmospheres to study in a self-consistent way the impact of photospheric inhomogeneities and correlated velocity fields on the formation of spectral lines and on spectroscopic abundance analyses. In fact, 3D simulations have been shown to be successful in reproducing several observational constraints such as the morphology of the granulation pattern in the Sun as well as detailed shapes of spectral lines in other solar-like stars. Recent analyses based on 3D simulations of convection at the surface of the Sun, dwarfs, subgiants and giants (e.g. Asplund 2005, Asplund and García Pérez 2001, Asplund et al 1999, Collet et al 2006, 2007) indicate that the structural differences between 3D hydrodynamical and 1D hydrostatic model atmospheres can significantly impact on the predicted strengths of spectral lines and, in turn, lead to large differences in the derived elemental abundances, especially at low metallicities. In the following, I will illustrate the main results of recent $3 \mathrm{D}$ simulations of convection at the surface of metal-poor red giants (Collet et al 2006, 2007) and discuss some applications of these models to spectral line formation and abundance analysis.

\section{The 3D convection simulations}

The 3D, time-dependent, compressible, explicit, radiative-hydrodynamical code by Stein and Nordlund (1998) has been used here to simulate convection at the surface of red giant stars with varying effective temperatures ( $\left.T_{\text {eff }} \approx 4700-5100 \mathrm{~K}\right)$, surface gravity $\log g=2.2(\mathrm{cgs})$, and metallicity ranging from solar down to $[\mathrm{Fe} / \mathrm{H}]=-3$. The equations of mass, momentum and energy conservation are solved on a discrete Cartesian mesh at moderate numerical resolution $(100 \times 100 \times 125)$ for a representative volume of stellar surface large enough to cover about ten granules horizontally and 11 pressure scale heights vertically. In terms of optical depth in the continuum at $\lambda=5000 \AA$, the simulations extend from $\log \tau_{5000} \approx-4$ to $\log \tau_{5000} \approx 7$. The upper and lower boundaries are open, whereas periodical boundary conditions are assumed horizontally. The simulations make use of realistic input physics: the equation of state comes from Mihalas et al (1988), continuous opacities from the Uppsala opacity package (updated version of Gustafsson et al 1975) and line opacities from Kurucz (1992) and Kurucz (1993). The solar chemical composition given by Grevesse and Sauval (1998) has been adopted with the abundances of all metals scaled proportionally to the $[\mathrm{Fe} / \mathrm{H}]$ value assumed for the simulation. The radiative heating term in the energy conservation equation is computed by solving the radiative transfer equation at each time step for all grid points at the surface and along eight inclined rays plus the vertical. To reduce the computational time, opacities are re-grouped in four opacity bins (Nordlund 1982). Also, local thermodynamic equilibrium (LTE) is assumed, with a Planckian source function and scattering treated as true absorption.

The temperature and density structures at the surface of red giant convection simulations are qualitatively fairly similar to the the ones previously described in the works by Asplund et al (1999) and Asplund and García Pérez (2001) on dwarfs and turnoff stars. Warm plasma rises from the stellar interior and rapidly cools as it approaches the optical surface; there it eventually becomes denser than the surrounding gas, turns over, and plunges back towards the interior. The bulk flows at the surface self-organize into a dynamical granulation pattern characterized by large, warm upflows separated by a network of cool, narrow downdrafts. Figure 1 shows the spatially resolved outgoing intensity in the continuum opacity bin for two red giant simulation snapshots with similar effective temperatures, at solar and very low $([\mathrm{Fe} / \mathrm{H}]=-3)$ metallicity. The properties of the emergent granulation patterns are slightly different in the two cases: at solar metallicity, the granules are typically larger and the overall intensity contrast is lower than at $[\mathrm{Fe} / \mathrm{H}]=-3$. More remarkable, however, is the metallicity dependence of the temperature stratification in the $3 \mathrm{D}$ simulations; figure 2 illustrates, as a function of optical depth, the predicted temperature structures from the two giant simulations at $[\mathrm{Fe} / \mathrm{H}]=0$ and $[\mathrm{Fe} / \mathrm{H}]=-3$. The resulting thermal structures are compared with the 1D stratifications from MARCS model atmospheres (Asplund et al 1997, Gustafsson et al 1975) generated for the same stellar parameters, and with the same opacity data and chemical 

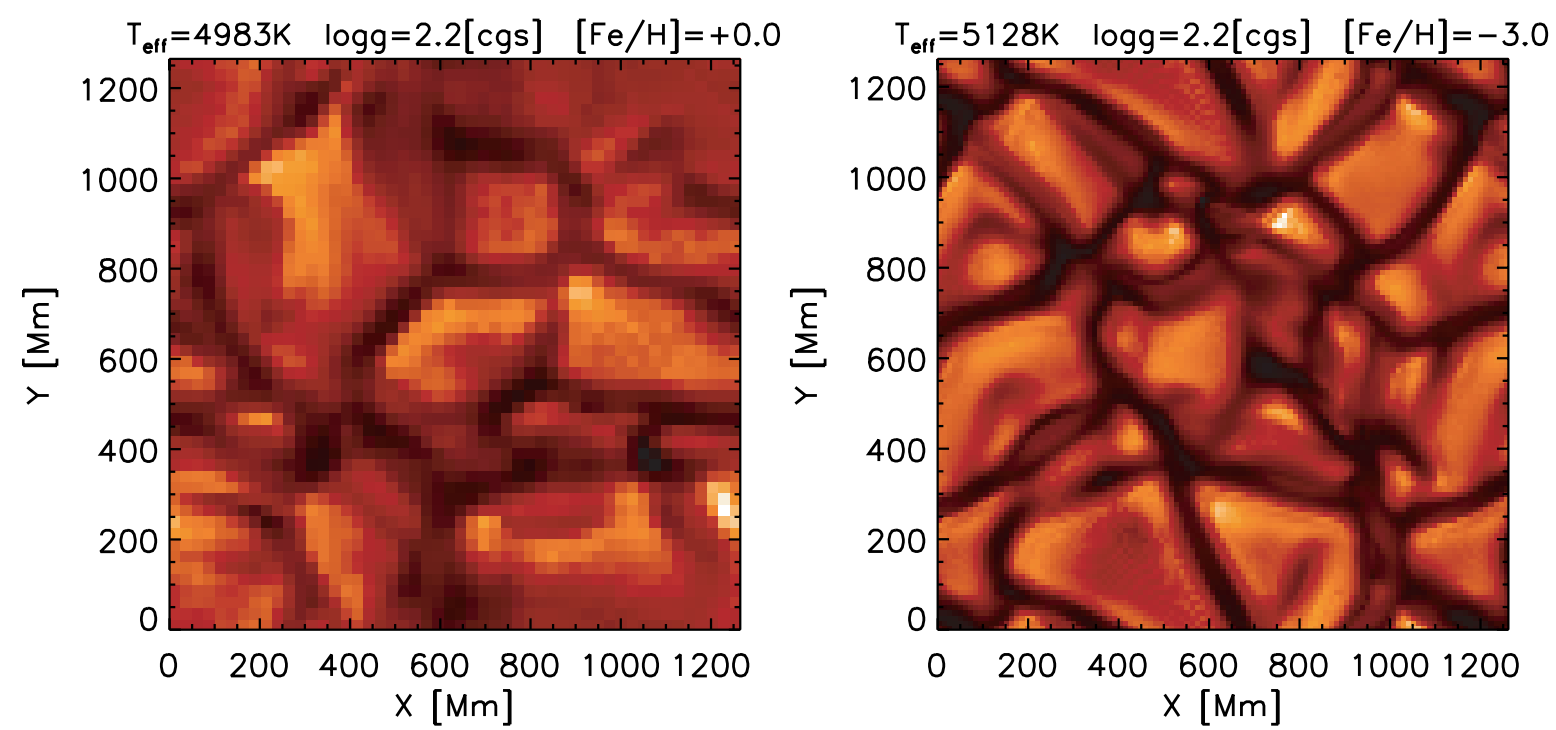

Figure 1. Spatially resolved emergent intensity in the continuum bin for two snapshots of 3D hydrodynamical simulations of red giants at $[\mathrm{Fe} / \mathrm{H}]=0.0($ left $)$ and $[\mathrm{Fe} / \mathrm{H}]=-3.0$ (right); the characteristic surface granulation pattern is apparent.
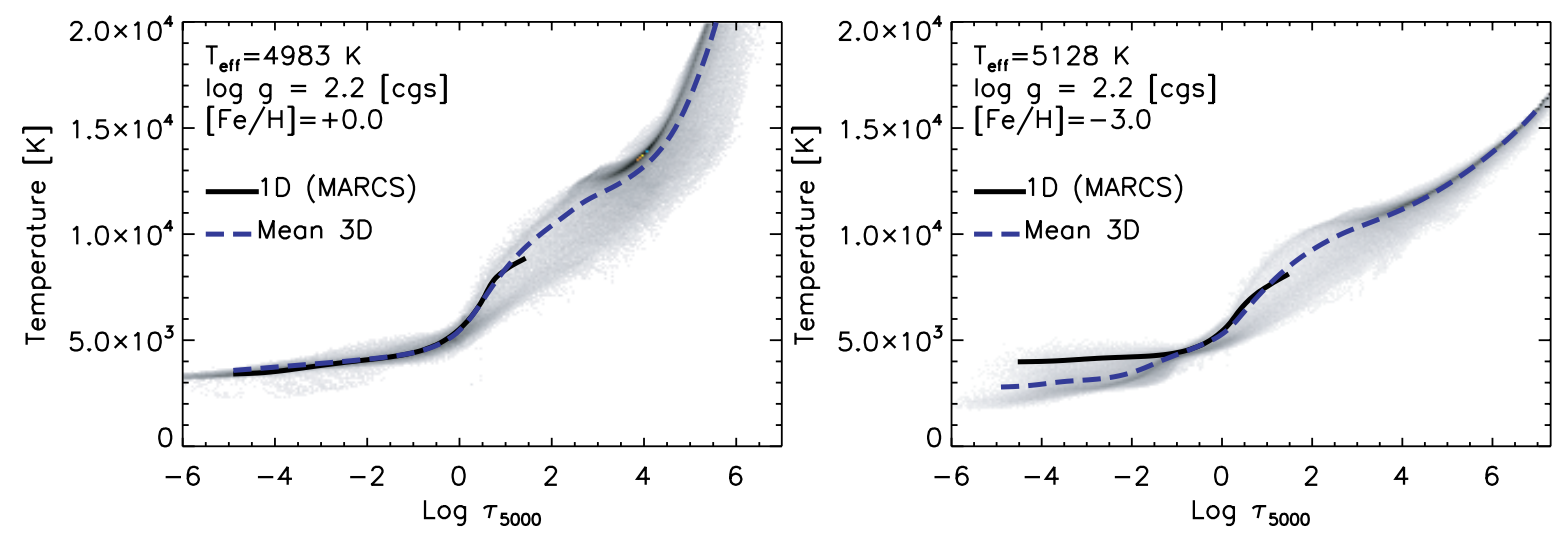

Figure 2. Temperature structure of two snapshots of $3 \mathrm{D}$ hydrodynamical simulations of red giants at $[\mathrm{Fe} / \mathrm{H}]=0.0$ (left) and $[\mathrm{Fe} / \mathrm{H}]=-3.0$ (right). Grey shaded area: temperature distribution as a function of optical depth in the continuum at $\lambda=5000 \AA$; darker areas indicate values with higher occurrence. Solid line: temperature stratification of a 1D MARCS model atmosphere computed for the same stellar parameters. Dashed line: mean temperature stratification of the 3D snapshot (temperature averages taken on surfaces of constant optical depth).

compositions as the 3D simulations. At solar metallicity, the mean temperature structure in the upper photosphere of the 3D simulation appears very similar to the stratification from the corresponding 1D model where radiative equilibrium is enforced by the stationary condition. At very low metallicity, in contrast, the temperature in the upper layers of the 3D simulations tends to remain significantly below the radiative equilibrium value marked by the corresponding $1 \mathrm{D}$ model. The reason for this difference can be found by looking at the energy conservation equation. The temperature in the upper layers of $3 \mathrm{D}$ simulations is mostly regulated by two competing mechanisms: radiative heating due to the reabsorption by spectral lines of radiation coming from deeper inside, and adiabatic cooling due to the expansion of the upflowing gas in granules. At very low metallicity, fewer and weaker lines contribute to the total opacity; therefore the significance of radiative heating from spectral lines is reduced, adiabatic cooling becomes preponderant, and the balance between the two mechanisms is achieved at lower temperatures than in stationary $1 \mathrm{D}$ models.
At $[\mathrm{Fe} / \mathrm{H}]=-3$, the temperature difference between the mean $3 \mathrm{D}$ and $1 \mathrm{D}$ photospheric structures is substantial and can amount to $\sim 1000 \mathrm{~K}$. With regard to stellar spectroscopy, such differences, as well as temperature and density inhomogeneities, can have a dramatic effect on the predicted strengths of spectral lines. The cooler photospheric stratification of the 3D convection simulations of very metal-poor stars is expected to significantly affect temperature-sensitive features, such as molecular lines or weak low-excitation lines from minority species (e.g. neutral metals). Gravity-sensitive features are also affected as the lower photospheric temperatures of the $3 \mathrm{D}$ simulations also imply reduced gas and electron pressures.

\section{Spectral line formation}

As mentioned above, 3D simulations of convection at the surface of late-type stars can be used as time-dependent, $3 \mathrm{D}$, hydrodynamical model atmospheres for spectral line formation purposes. In this section, I will sketch the 
general strategy used to compute line profiles with 3D model atmospheres and to derive differential 3D-1D elemental abundances. From the full convection simulations, representative sequences of typically $\sim 50-100$ snapshots are taken at regular intervals in time; these time series should cover sufficiently long periods to provide good statistical samples of the evolution of the surface granulation patterns. The snapshots are interpolated to a finer depth scale to increase the spatial resolution in the atmospheric layers with optical depth less than $\log \tau_{5000} \approx 2.5$ to achieve higher numerical accuracy. Flux profiles are computed for a variety of lines from metals and molecules. The radiative transfer equation is solved for about 60-100 wavelength points per spectral line profile, along typically $\sim 10-30$ rays, and for all grid points at the surface of the 3D model. As during the construction of the simulation, the assumption of LTE is made, with scattering treated as true absorption. The same numerical code, input physics and opacity data are used to compute spectral line profiles with 1D model atmospheres corresponding to the same stellar parameters; in the $1 \mathrm{D}$ calculations, a nonzero value of the micro-turbulence is adopted to mimic non-thermal Doppler broadening of the lines. I would like to emphasize once more here that in $3 \mathrm{D}$, in contrast, no free parameters such as micro- or macro-turbulence enter the calculations: only the velocity fields predicted by the convection simulations are taken into account to reproduce non-thermal broadening, asymmetries and wavelength shifts associated with the bulk motions of the gas in the stellar atmosphere.

The impact of 3D hydrodynamical models on stellar spectroscopy is evaluated by means of a differential 3D-1D abundance analysis based on a simple curve-of-growth method. The abundance of the trace element is varied independently in the $3 \mathrm{D}$ and $1 \mathrm{D}$ calculations to match the equivalent width of a given spectral line; the difference between the two abundances represents the differential 3D-1D correction to the abundance of the trace element derived from that particular spectral feature.

\section{Results}

In this section, I will present some representative results of differential 3D-1D LTE abundance analyses at very low metallicity, where the impact of 3D models on the strengths of spectral lines is anticipated to be largest. Figure 3 shows the differential 3D-1D LTE corrections to the Fe abundance for a very metal-poor red giant as derived from 'fictitious' Fe I lines at $\lambda=5000 \AA$ with varying equivalent widths $\left(W_{\lambda}\right.$ between 10 and $80 \mathrm{~m} \AA$ ) and lower-level excitation potentials ${ }^{2}$. Such corrections are overall large and negative, particularly in the case of low-excitation lines, for which the differences between the predicted 3D and 1D LTE Fe abundances are of the order of -1 dex. The behaviour of the differential 3D-1D LTE iron abundances from Fe I lines at low metallicity can be qualitatively explained by comparing the variations of the

2 Fictitious lines (Asplund 2005, Collet et al 2006, 2007, Steffen and Holweger 2002) provide a benchmark to analyse the behaviour of spectral lines solely as a function of lower-level excitation potential, wavelength and strength, disregarding complications introduced by blends and wavelength dependency of continuous opacities in samples of real lines.

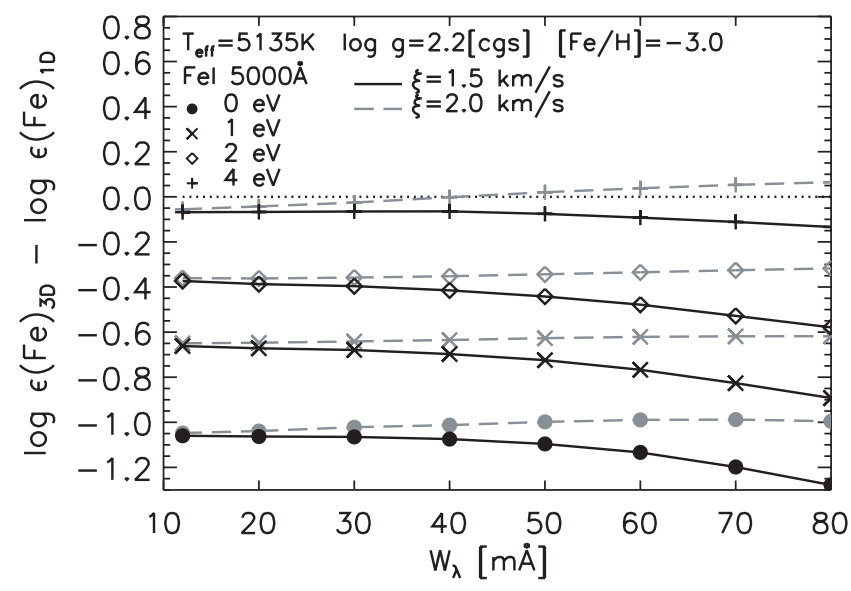

Figure 3. Differences between the Fe abundances derived in LTE from 'fictitious' Fe I lines at $\lambda=5000 \AA$ with a 3D hydrodynamical and a $1 \mathrm{D}$ MARCS model atmosphere of very metal-poor red giant. The differential abundances are plotted as a function of equivalent width $W_{\lambda}$ and lower-level excitation potential of the lines, and for two different choices of the micro-turbulence parameter $\xi$ in the 1D calculations.

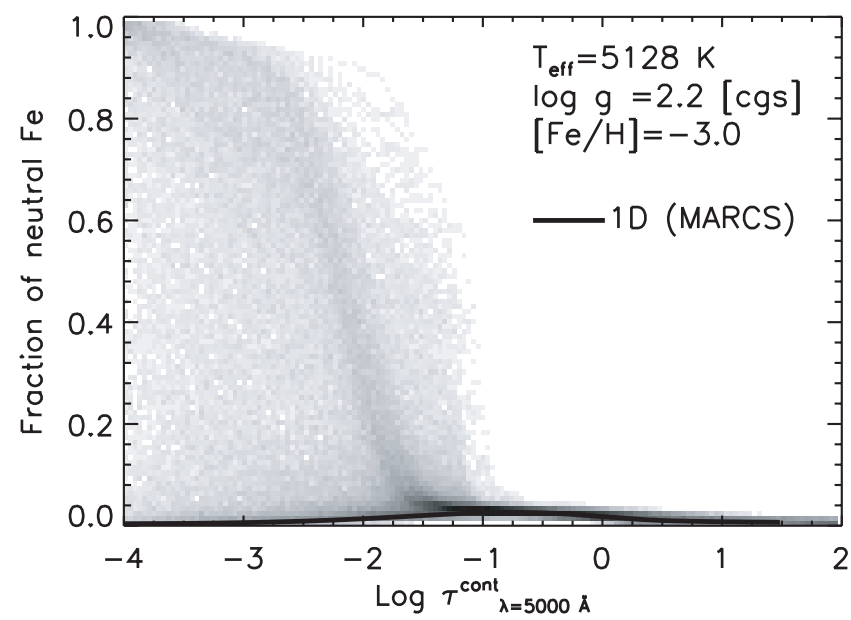

Figure 4. Ratio of neutral to total iron number densities $\left(n_{\mathrm{FeI}} / n_{\mathrm{FeI}}\right)$ as a function of optical depth in the continuum at $5000 \AA$ in the atmosphere of a red giant star at $[\mathrm{Fe} / \mathrm{H}]=-3$. Grey shaded area: distribution of $n_{\mathrm{FeI}} / n_{\mathrm{FeI}}$ values predicted with the use of a $3 \mathrm{D}$ model atmosphere: darker areas indicate values with higher occurrence. Over-plotted is the fraction of neutral iron as a function of optical depth in the corresponding 1D MARCS model atmosphere (solid line).

fraction of neutral-to-total Fe number densities with optical depth in the 3D hydrodynamical and in the corresponding 1D MARCS model atmospheres (figure 4). Under the assumption of LTE, in the 1D MARCS model, iron is nearly completely ionized throughout the atmosphere; in contrast, in the $3 \mathrm{D}$ model, the lower surface temperatures encountered in the upper photosphere allow a significant fraction of iron to recombine into neutral form. Hence, at a given Fe abundance, the density of absorbing neutral iron particles will be higher in the upper photosphere of the 3D model than in the 1D model and $\mathrm{Fe} \mathrm{I}$ lines will also appear stronger. Compared with the $1 \mathrm{D}$ case, a lower Fe abundance is therefore required in $3 \mathrm{D}$ to match the same equivalent width of a given $\mathrm{Fe} I$ line.

A similar behaviour holds for the differential 3D-1D LTE abundance corrections derived from lines of other neutral 
metals (Asplund 2005, Collet et al 2007). Molecule formation as well shows an extreme sensitivity to temperature in the upper photospheric layers of late-type stars. This temperature sensitivity causes the 3D-1D LTE corrections to elemental abundances derived from the analysis of molecular features to also be large and negative in very metal-poor stars (Asplund and García Pérez 2001, Collet et al 2006, 2007).

\section{3D abundance analysis of extreme halo stars}

An interesting application of 3D model atmospheres of very metal-poor stars is the investigation of the impact of stellar granulation on the abundance analysis of the two extremely iron-poor halo stars HE0107-5240 and HE1327-2326 (Christlieb et al 2002, Frebel et al 2005). These two stars are remarkable in the sense that the abundance of the iron-peak elements in their atmospheres is the lowest ever observed in stellar environments $([\mathrm{Fe} / \mathrm{H}]<-5)$, while at the same time they are characterized by very large overabundances of carbon, nitrogen and oxygen with respect to iron. The interest aroused by these two objects comes from the consideration that HE0107-5240 and HE1327-2326 might be direct descendants of a previous generation of metal-free stars. Various hypotheses have been proposed to explain the origin of the two stars but in order to identify the most plausible formation scenario, an accurate determination of their chemical composition is necessary. Collet et al (2006) and Frebel et al (2008) have performed an abundance analysis of HE0107-5240 and HE1327-2326 with the aid of 3D model atmospheres and following the basic procedure sketched in section 3. The main results of the 3D-1D LTE abundance analysis of the two stars are summarized in table 1. The 3D LTE Fe abundance is estimated to be about -0.2 dex lower than that according to the $1 \mathrm{D}$ analysis, whereas the abundances of carbon, nitrogen and oxygen derived from $\mathrm{CH}, \mathrm{NH}$ and $\mathrm{OH}$ molecular lines are decreased by -0.7 dex or more. In the case of HE0107-5240, it is possible to derive the value of $\mathrm{C}$ and $\mathrm{N}$ abundances from more than one molecular indicator. Interestingly, while Christlieb et al (2004) found a discrepancy of about 0.3 dex between the 1D carbon abundance values derived from $\mathrm{CH}$ and $\mathrm{C}_{2}$ lines, the $3 \mathrm{D}$ analysis brings the abundances from these two indicators down to the same value of $\log \epsilon(\mathrm{C}) \approx 5.7 \mathrm{dex}$. The $3 \mathrm{D}$ analysis of $\mathrm{CN}$ lines in HE0107-5240, however, returns an extremely large and negative $3 \mathrm{D}-1 \mathrm{D}$ correction to the nitrogen abundance (about $-2 \mathrm{dex}$ ), which is not consistent with the 3D-1D correction evaluated from NH lines (see table 1). This discrepancy could be, on the other hand, ascribable to the highly uncertain $g f$-values of NH lines (Collet et al 2006). This problem certainly deserves further investigation.

\section{Discussion}

The prediction of photospheric temperatures significantly below the radiative equilibrium value is a central result of surface convection simulations of metal-poor late-type stars. One implication of this result is that 3D-1D abundance corrections in very metal-poor stars are in general significantly larger than other systematic errors usually quoted
Table 1. Average differential 3D-1D LTE corrections to the C, N and $\mathrm{O}$ abundances in the extremely iron-poor halo stars HE0107-5240 and HE1327-2326 as derived from low-excitation molecular lines; average corrections to the Fe abundance estimated from $\mathrm{Fe}$ I lines are also given. Note: abundances are expressed in the customary logarithmic scale where $\log \epsilon(\mathrm{H})=12$.

\begin{tabular}{lclllcc}
\hline & & \multicolumn{2}{c}{ HE0107-5240 } & & \multicolumn{2}{c}{ HE1327-2326 } \\
\cline { 3 - 4 } \cline { 6 - 7 } Element & Indicator & $\log \epsilon_{1 \mathrm{D}}^{\mathrm{a}}$ & $\log \epsilon_{3 \mathrm{D}}^{\mathrm{b}}$ & & $\log \epsilon_{1 \mathrm{D}}^{\mathrm{c}}$ & $\log \epsilon_{3 \mathrm{D}}^{\mathrm{c}}$ \\
\hline $\mathrm{C}$ & $\mathrm{CH}$ & 6.81 & 5.75 & & 6.90 & 6.21 \\
$\mathrm{C}$ & $\mathrm{C}_{2}$ & 7.11 & 5.7 & & - & - \\
$\mathrm{N}$ & $\mathrm{NH}$ & 4.83 & 3.8 & 6.79 & 6.10 \\
$\mathrm{~N}$ & $\mathrm{CN}$ & $5.22(4.93)^{\mathrm{d}}$ & 3.2 & & - & - \\
$\mathrm{O}$ & $\mathrm{OH}$ & 5.66 & 5.0 & & 6.84 & 6.12 \\
$\mathrm{Fe}$ & $\mathrm{Fe} \mathrm{I}$ & 2.06 & 1.8 & & 1.79 & 1.5 \\
\hline
\end{tabular}

${ }^{a}$ Bessell et al (2004), Bessell and Christlieb (2005), Christlieb et al (2004).

${ }^{\mathrm{b}}$ Collet et al (2006).

${ }^{\mathrm{c}}$ Frebel et al (2008).

${ }^{\mathrm{d}}$ Assuming $\log \epsilon_{1 \mathrm{D}}(\mathrm{C})=6.81(7.11)$.

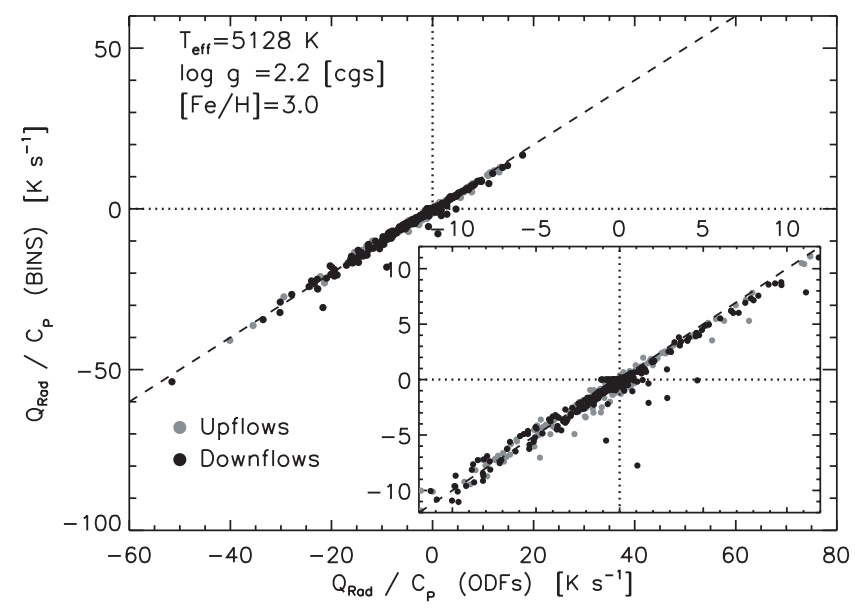

Figure 5. Comparison between the radiative heating rates computed in '1.5D' approximation for all grid points on a vertical slice of a simulation snapshot of very metal-poor red giant using the opacity-binning scheme ('BINS') and monochromatic radiative transfer with opacity distribution functions ('ODFs'). The radiative heating rates per unit mass $\left(Q_{\text {rad }}\right)$ are normalized with respect to the specific heat (per unit mass) at constant pressure $\left(C_{\mathrm{P}}\right)$. A magnified view of the plot in the region of low radiative heating rates is shown in the small panel.

in classical abundance analyses (Gustafsson 2004). It is therefore necessary to investigate to what extent the cooler temperature stratification of the 3D models is controlled by the assumptions made during the construction of the simulations.

A relevant question in this respect is whether the opacity-binning scheme used for the calculation of the radiative heating rates represents a reasonably accurate approximation to the solution of the radiative transfer equation for the full set of wavelengths of the original opacity distribution functions (ODFs). Figure 5 shows the results of test calculations where the radiative heating rates are computed with the two different approaches in ' $1.5 \mathrm{D}$ ' approximation along individual columns in a vertical slice of a metal-poor red giant simulation snapshot. The correlation between the opacity-binning and ODF-based schemes is very good, suggesting that the former is probably accurate enough 


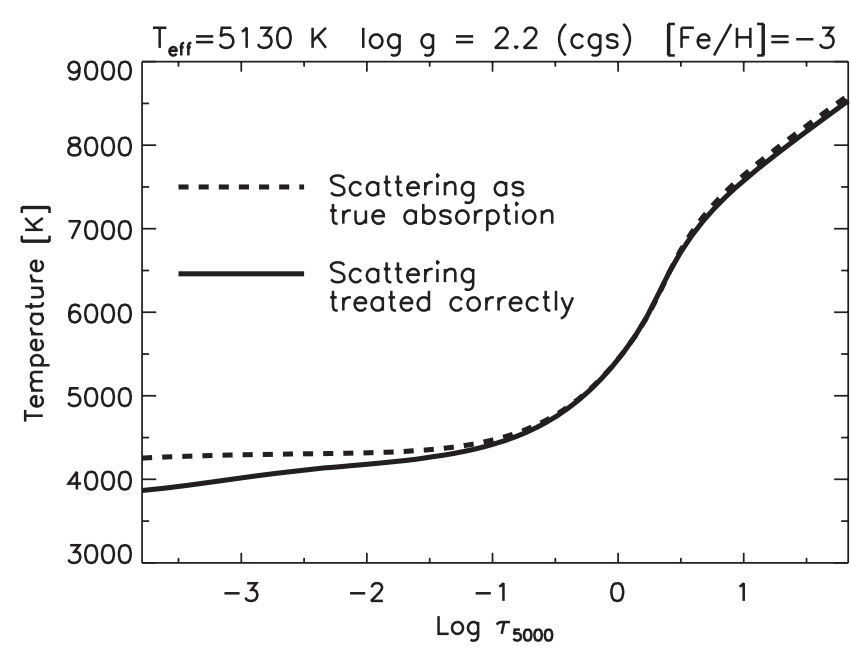

Figure 6. Comparison between the temperature stratifications of two OS-MARCS model metal-poor giant atmospheres computed for the same stellar parameters but with two different implementations of continuous scattering. Solid line: scattering correctly treated as such in the source function (standard version of OS-MARCS); dashed line: scattering included as true absorption, as in the 3D simulations presented here.

in reproducing radiative heating and temperatures at the surface of metal-poor 3D simulations.

Another important issue is whether the treatment of scattering as true absorption in the solution of the radiative transfer equation introduces systematic errors in the temperature at the surface of $3 \mathrm{D}$ convection simulations. According to preliminary tests with 1D OS-MARCS (Gustafsson et al 2008) model atmospheres of metal-poor giants, the 1D temperature stratifications predicted when scattering is included as true absorption is overall hotter than in calculations where scattering is treated correctly as such. In the example considered in figure 6 , temperature differences between the two cases reach $300 \mathrm{~K}$ in the upper photosphere. This result would therefore suggest that the radiative heating rates and temperature might actually be overestimated at the surface of 3D models of metal-poor stars. It is not obvious, however, that the effect of implementing scattering as true absorption would proceed in the same direction in 3D. A full 3D treatment of scattering is paramount in this respect to establish whether the surface temperatures are possibly underestimated in the present metal-poor 3D models. Refer to the contribution of $\mathrm{W}$ Hayek in these proceedings for more details about $3 \mathrm{D}$ radiative transfer with scattering in convection simulations (Hayek 2008).

It is also crucial to assess how spectral line formation is affected by the approximations involved in the solution of the radiative transfer equation. The treatment of scattering as true absorption is a matter of concern also for $3 \mathrm{D}$ line formation calculations. Rayleigh scattering of $\mathrm{H} \mathrm{I}_{\mathrm{I}}$ is, in fact, an important source of extinction in the UV and blue part of the spectrum. At those wavelengths, implementing scattering as true absorption causes the outgoing flux in the continuum to be underestimated and hence leads to the prediction of weaker spectral lines. The effect is expected to be more important at very low metallicities, due to the weak line-blocking, and in metal-poor 3D model atmospheres, where the density of scatterers ( $\mathrm{H} \mathrm{I}$ ) is high because of the low surface temperatures. The use of a differential 3D-1D
Table 2. Non-LTE Fe abundances derived for the extreme halo stars HE0107-5240 and HE1327-2326 using 1D MARCS models and the mean temperature stratification versus optical depth from 3D models. The non-LTE calculations assume the model Fe atom by Collet et al (2005), efficient Drawin-like H + Fe collisions (Drawin 1968, 1969) and thermalization of the uppermost Fe I levels.

\begin{tabular}{lcclcc}
\hline & \multicolumn{2}{c}{ HE0107-5240 } & & \multicolumn{2}{c}{ HE1327-2326 } \\
\cline { 2 - 3 } \cline { 5 - 6 } Model & {$[\mathrm{Fe} / \mathrm{H}]_{\text {LTE }}$} & {$[\mathrm{Fe} / \mathrm{H}]_{\text {non-LTE }}$} & & {$[\mathrm{Fe} / \mathrm{H}]_{\text {LTE }}$} & {$[\mathrm{Fe} / \mathrm{H}]_{\text {non-LTE }}$} \\
\hline 1D MARCS & -5.40 & -4.65 & & -5.70 & -5.15 \\
Mean 3D & -5.60 & -4.75 & & -5.95 & -5.05 \\
\hline
\end{tabular}

abundance analysis ensures at least that the uncertainties in the treatment of scattering are minimized.

Finally, I would like to caution that many of the abundance indicators considered in the present paper (e.g. neutral metals and possibly molecules as well) are most likely affected by departures from LTE or from chemical equilibrium at the local temperature. Neutral iron, for instance, is expected to be prone to such departures. The main non-LTE mechanism for $\mathrm{Fe}_{\mathrm{I}}$ in the photospheres of late-type stars is efficient overionization driven by the UV radiation field coming from layers deeper inside. This causes $\mathrm{Fe}$ I levels to be underpopulated with respect to LTE, makes Fe I lines weaker than the LTE case at a given Fe abundance and, consequently, leads to the prediction of higher Fe abundances. Fe I departures from LTE have been estimated by Collet et al (2006) for the two extreme halo stars HE0107-5240 and HE1327-2326 by means of a 1D analysis (table 2). The results of these preliminary calculations clearly suggest that the non-LTE effects are considerable and opposite to the corrections for stellar granulation. This implies that a combined 3D non-LTE analysis of $\mathrm{Fe} I$ line formation in a similar way as done by Asplund et al (2003) and Barklem et al (2003) for Li I is essential for producing realistic Fe abundance determinations. For completeness, I should also add that the departures of Fe I from LTE depend crucially on the magnitude of $\mathrm{H}+\mathrm{Fe}$ collisional excitation and ionization processes, whose cross-sections are still poorly known. This explains, at least in part, why current estimates of non-LTE effects on Fe I line formation are still hampered by large uncertainties (see also the review by A Korn in these proceedings (Korn 2008)).

\section{Conclusions}

I have presented here some illustrative results on the application of 3D surface convection simulations of very metal-poor stars to spectral line formation in LTE. The differences between the predicted temperature stratifications of 3D hydrodynamical simulations and 1D hydrostatic model atmospheres, and the 3D temperature and density inhomogeneities and correlated velocity gradients can significantly affect line strengths and, in turn, the elemental abundances inferred from spectral lines. At very low metallicities, the deviations of the mean 3D thermal structure from the classical 1D stratification are largest, and cause the 3D-1D LTE differential abundances to be negative and considerable for lines of neutral species (down to about -1 dex for weak low-excitation Fe I line). Corrections to 
carbon, nitrogen and oxygen (CNO) abundances derived from weak low-excitation $\mathrm{CH}, \mathrm{NH}$ and $\mathrm{OH}$ features are also found to be typically in the range -0.5 to -1.0 dex in very metal-poor giants (Collet et al 2007). Finally, I have discussed possible systematic errors affecting the present $3 \mathrm{D}$ abundance analyses, such as departures of Fe I line formation from LTE; non-LTE corrections to the Fe abundance are opposite to and, according to preliminary $1 \mathrm{D}$ test calculations, of the same order of magnitude as the ones due to stellar granulation.

\section{Appendix. Discussion}

Q: (Rainer Wehrse) When you mentioned ODFs, did you refer to the classical ODFs for static media or generalized ODFs that take velocity gradients into account?

A: The ODFs used here for the construction of the opacity bins are the 'classical' ones computed for static media which do not account indeed for vertical velocity gradients.

Q: (Andreas Korn) We know that the peculiar CNO abundances of the ultra-metal-poor stars have an effect on the $T-\tau$ relation in 1D. Do you consider the feedback from non-solar abundance ratios on your $3 \mathrm{D}$ model in the opacity-binning approach?

A: There is no feedback on the $T-\tau$ relations here, in the sense that the $3 \mathrm{D}$ and $1 \mathrm{D}$ models are not re-computed taking into account the particular chemical abundance pattern derived for these stars. Let me add here, however, that the 3D-1D abundance analysis is differential, and both $3 \mathrm{D}$ and $1 \mathrm{D}$ models have been constructed with identical composition, that is, scaled solar with $[\mathrm{Fe} / \mathrm{H}]=-3$; the physical structure of a $1 \mathrm{D}$ model atmosphere of HE0107-5240 computed assuming the detailed abundance pattern of this star closely resembles the structure of a 1D model atmosphere constructed for a scaled solar composition with $[\mathrm{Fe} / \mathrm{H}]=-3$. For the line formation calculations and abundance analysis, on the other hand, we do take into account the actual chemical composition of these stars to compute chemical equilibrium and background opacities.

Q: (Hans-Günther Ludwig) A technical question: Is the horizontal jitter visible in the movie of the metal-poor giant intrinsic to the model or a mere artifact of 'movie production'?

A: The 'jitter' in the animated time sequence is not real and it is indeed just an artifact introduced by the procedure used to make the movie.

Q: (Bertrand Plez) Is there a way for you to check the impact of the horizontal temperature fluctuations in the 3D model on abundances in non-LTE, compared with the $\langle 3 \mathrm{D}\rangle-1 \mathrm{D}$ effect?

A: Essentially, the only correct way of estimating this is to actually solve the full $3 \mathrm{D}$ non-LTE line formation problem, which for Fe I is still extremely demanding. It is not obvious whether one could obtain at least an approximate answer in the right direction with some sort of $1.5 \mathrm{D}$ calculation.

Q: (Robert Kurucz) 3D models have systematic errors because of inadequate treatment of opacity. You can estimate this error by computing a $1 \mathrm{D}$ model with the same opacity treatment and comparing it with a state-of-the-art 1D model: the temperature distributions will be different.

A: I agree: a more correct way to compute the differential $3 \mathrm{D}-1 \mathrm{D}$ abundance corrections is indeed to use 1D models for which the radiative transfer is solved with the same opacity-binning technique adopted in the $3 \mathrm{D}$ models. At the moment, we only estimate the error in our procedure in an approximate way by performing the differential analysis with different 1D models (e.g. MARCS and ATLAS9): at $[\mathrm{Fe} / \mathrm{H}]=-3$, the deviation in terms of abundance determinations is typically of the order of 0.05-0.1 dex depending on which lines are used.

\section{References}

Asplund M 2005 Annu. Rev. Astron. Astrophys. 43 481-530 Asplund M and García Pérez A E 2001 Astron. Astrophys. 372 601-15

Asplund M, Gustafsson B, Kiselman D and Eriksson K 1997 Astron. Astrophys. 318 521-34

Asplund M, Nordlund A, Trampedach R and Stein R F 1999 Astron. Astrophys. 346 L17-20

Asplund M, Carlsson M and Botnen A V 2003 Astron. Astrophys. 399 L $31-4$

Baade W 1944 Astrophys. J. 100137

Barklem P S, Belyaev A K and Asplund M 2003 Astron. Astrophys. 409 L1-4

Bessell M S and Christlieb N 2005 From Lithium to Uranium: Elemental Tracers of Early Cosmic Evolution ed V Hill, P François and F Primas (IAU Symposium 228) pp 237-8

Bessell M S, Christlieb N and Gustafsson B 2004 Astrophys. J. Lett. 612 L61-3

Böhm-Vitense E 1958 Z. Astrophy. 46108

Canuto V M and Mazzitelli I 1991 Astrophys. J. 370 295-311

Carlsson M, Stein R F, Nordlund A and Scharmer G B 2004 Astrophys. J. Lett. 610 L137-40

Chamberlain J W and Aller L H 1951 Astrophys. J. 11452

Christlieb N, Bessell M S, Beers T C, Gustafsson B, Korn A, Barklem P S, Karlsson T, Mizuno-Wiedner M and Rossi S 2002 Nature 419 904-6

Christlieb N, Gustafsson B, Korn A J, Barklem P S, Beers T C, Bessell M S, Karlsson T and Mizuno-Wiedner M 2004 Astrophys. J. 603 708-28

Collet R, Asplund M and Thévenin F 2005 Astron. Astrophys. 442 643-50

Collet R, Asplund M and Trampedach R 2006 Astrophys. J. Lett. 644 L121-4

Collet R, Asplund M and Trampedach R 2007 Astron. Astrophys. $469687-706$

Drawin H W 1968 Z. Phys. 211 404-17

Drawin H W 1969 Z. Phys. 225 483-93

Frebel A et al 2005 Nature 434 871-3

Frebel A, Collet R, Eriksson K, Christlieb N and Aoki W 2008 Astrophys. J. 684 588-602

Freytag B, Steffen M and Dorch B 2002 Astron. Nachr. 323 213-9

Grevesse N and Sauval A J 1998 Space Sci. Rev. 85 161-74

Gustafsson B 2004 Origin and Evolution of the Elements ed A McWilliam and M Rauch (Cambridge: Cambridge University Press) $\mathrm{p} 102$

Gustafsson B and Jorgensen U G 1994 Astron. Astrophys. Rev. 6 19-65

Gustafsson B, Bell R A, Eriksson K and Nordlund Å 1975 Astron. Astrophys. 42 407-32

Gustafsson B, Edvardsson B, Eriksson K, Graae Jorgensen U, Nordlund A and Plez B 2008 Astron. Astrophys. 486 951-70

Hayek W 2008 Phys. Scr. T133 014006

Korn A 2008 Phys. Scr. T133 014009 
Krishna Swamy K S 1969 Astron. Astrophys. 1 297-304

Kurucz R L 1992 Rev. Mexicana Astron. Astrofis. 23 181-6

Kurucz R L 1993 Opacities for Stellar Atmospheres (Kurucz $C D$-ROMs vol 2-12) (Cambridge, MA: SAO)

Ludwig H G, Allard F and Hauschildt P H 2002 Astron. Astrophys. 395 99-115

Mihalas D, Däppen W and Hummer D G 1988 Astrophys. $J$. $331815-25$
Nordlund Å 1982 Astron. Astrophys. 107 1-10

Nordlund $\AA$ and Dravins D 1990 Astron. Astrophys. 228 155-217

Roman N G 1950 Astrophys. J. 112554

Schwarzschild M and Schwarzschild B 1950 Astrophys. J. 112248

Steffen M and Holweger H 2002 Astron. Astrophys. 387 258-70

Stein R F and Nordlund A 1998 Astrophys. J. 499 914-33

Vögler A 2004 Astron. Astrophys. 421 755-62 